\title{
SLC changes its tactics
}

\section{San Francisco}

AFTER a short-lived day in the sun during which it became the world's leading producer of elusive $Z^{0}$ particles, the Stanford Linear Collider (SLC) is now preparing to change tactics in order to avoid direct competition with a more powerful machine gearing up to speed at CERN, the 14-nation European scientific consortium based in Geneva.

For the SLC, which currently produces about $10 Z^{\prime \prime}$ s a day, the change has come too soon. By studying the $Z^{\prime \prime}$, one of the fundamental particles of the unified electroweak force, physicists will be able to pin down some of the poorly known parameters in their 'standard model' of elementary particles and forces. The original plan called for up to two years of dominance before the rival Large ElectronPositron (LEP) collider started up at CERN. Indeed, in the past few weeks, SLC scientists have announced the world's most accurate value for the mass of the $Z^{0}$, as well as a new limit on the number of 'families' - sets of related quarks and leptons - into which elementary particles are classified.

But nagging technical difficulties have put the collider far behind schedule and dramatically reduced its time at the top.

Earlier this month, the more powerful LEP made its first $Z^{0}$ particles (see Nature 340, 495; 17 August 1989), signalling a new era for the SLC. Once the European collider concludes its pilot run and is refined for full-scale efforts around the end of September, its California counter-. part will have to relinquish its leadership role and instead try to fill niches. "It just really depends on how quickly CERN works its bugs out. After approximately October 1, it's going to be difficult to compete on a particle-by-particle basis", said SLC spokesman Michael Riordan. "We have to do special things that the nature of our detector allows us to do."

The first tactic involves electron beams, which collide to produce the $Z^{0}$ particles. While the LEP beams come together at a much higher rate than SLC's, and consequently produce many times more particles, the California facility employs a narrower beam that offers its own advantages. Because the particles the $Z^{0}$ decays into are extremely short-lived - lasting on the order of a trillionth of a second - many of them will decay while still within the wider beam used at CERN. Consequently, the LEP physicists will not be able to tell which came directly from $Z^{0}$ s and which are secondary creations, according to Riordan. The detection equipment needed to make such determinations should be installed at the SLC in early October.

For the longer term, beginning perhaps in 1990 , the SLC will use polarized electron beams, in which the electron spins are aligned with respect to the beam axis. By giving SLC physicists an extra degree of control over reactions that make $Z^{\prime \prime}$, polarized beams permit the same level of accuracy to be achieved on certain measurements with 10 times fewer $Z^{\prime \prime}$ s than
CERN. In measuring the Weinberg angle, the basic parameter of electroweak unification, SLC and CERN can thus compete on even terms.

In recent weeks, news accounts have focused on the well-known competition between the two facilities. By switching tactics, the SLC group hopes to play down the rivalry with CERN and emphasize ways for the two efforts to complement each other.

Robert Buderi

\section{EDUCATION REFORM}

\section{UK university charters stay the axe}

\section{London}

THE resolution last week of a dispute over academic tenure at Aston University in Birmingham indicates that half of Britain's universities are still unable to dismiss tenured staff almost one year after the new education reform act ruled that they could.

Aston University wanted to impose compulsory retirement on 12 members of staff in the department of engineering. In response, the Association of University Teachers (AUT) threatened to take out an injunction to prevent the dismissals. The ensuing legal battle ended when the vicechancellor of the supreme court, Nicholas Browne-Wilkinson, who was appointed to resolve the dispute, ruled that Aston's charter and statutes protect academic staff from compulsory redundancy unless the university has "good cause" to dismiss them. More than half of Britain's universities have similar charters.

Many universities are attempting to reduce staffing levels by voluntary redundancies and retirements to compensate for financial cutbacks and falling student numbers in certain fields. But some universities are being forced to try more drastic steps to reduce costs. The new Education Reform Act allows the dismissal of tenured staff, but the charters under which the universities operate must first be rewritten. Five senior academics and lawyers have been asked by the Department of Education to look at individual charters to amend the clauses that guarantee tenure.

The new legislation is not retrospective, however, and any academic appointed or promoted before 20 November 1987, when the bill was published, will still be protected. David Packham, Aston's secretary and registrar, said that this was a serious flaw in the legislation and that some universities might suffer serious financial problems because they are unable to shed the academic posts they cannot afford. "The answer for some may be bankruptcy to save themselves and to worry about the consequences later," he said.

Ben Webb

\section{Indian launch vehicle grounded}

\section{New Delhi}

INDIA's Augmented Satellite Launch Vehicle (ASIV) has been grounded on the advice of an expert review panel after two successive failures. The panel has asked the Indian Space Research Organisation (ISRO) to modify the design of the rocket before attempting another flight. According to ISRO, the third ASLV flight incorporating the suggested changes will not take place for a year or more.

The panel suggested the design changes after a detailed investigation of the crash of ASLV during its second flight in July 1988. It concluded that ASLV is highly unstable (because of its high length-todiameter ratio of 23) and that its control system is inadequate, particularly during its flight in the lower atmosphere where dynamic pressure is high. No control of the rocket is available during the critical phase between the burn-out of the two strap-on booster motors and the ignition of the first-stage motor. During the abortive ASLV flight, the boosters burnt out a little too soon and the period of "no control" lasted for four seconds, during which the yaw angle rapidly increased. The increased load caused the vehicle to break up. These design inadequacies were not revealed in the first flight on $24 \mathrm{March}$ 1987 , as its failure was due to non-ignition of the first-stage motor.

ISRO has been asked to redesign the auto-pilot system and strap-on boosters, enhance the control margin and confirm them through additional simulation and destructive structural tests. All this work will be done by a new interdisciplinary design group. ISRO has admitted that the original design of ASLV failed to recognize the full impact of inadequate control during the critical phase flight or that of wind shears and gusts on the structural margins of safety.

ASLV is expected to be ISRO's workhorse for placing payloads up to 150 kilograms. But lessons learnt from it have already led to design modifications in the Polar Satellite Launch Vehicle (PSLV) which will place one-tonne payloads in polar orbit.
K. S. Jayaraman 